\title{
Ion irradiation-induced crystal structure changes in inverse
}

\section{spinel $\mathrm{MgIn}_{2} \mathrm{O}_{4}$}

Ming Tang ${ }^{1}$, James A. Valdez ${ }^{1}$, Yongqiang Wang ${ }^{1}$, Jian Zhang ${ }^{1,2}$, Blas P. Uberuaga ${ }^{1}$, Kurt E. Sickafus $^{3}$

${ }^{1}$ Materials Science and Technology Division, Los Alamos National Laboratory,

Los Alamos, New Mexico 87545, USA

${ }^{2}$ College of Energy, Xiamen University, Xiamen, Fujian 361005, China

${ }^{3}$ Department of Materials Science and Engineering, University of Tennessee

Knoxville, TN 37996, USA

\begin{abstract}
$400 \mathrm{keV} \mathrm{Ne}$ and $200 \mathrm{keV} \mathrm{He}$ ion irradiations were performed on fully inverse $\mathrm{MgIn}_{2} \mathrm{O}_{4} \operatorname{samples}$ at cryogenic temperature $(\sim 77 \mathrm{~K})$, in order to examine the influence of radiation-induced cation disordering on crystal structure. In the case of $\mathrm{MgIn}_{2} \mathrm{O}_{4}$ samples irradiated with $\mathrm{Ne}$ ions to a peak displacement damage dose of 4 displacements per atom (dpa), a spinel-to-rocksalt phase transformation was observed. By contrast, for $\mathrm{MgIn}_{2} \mathrm{O}_{4}$ samples irradiated with He ions to a peak displacement damage dose of $5 \mathrm{dpa}$, the only observed structural effect involved cation rearrangements from an inverse to a "random" spinel structure.
\end{abstract}


The crystal structure of spinel with the general formula $\mathrm{AB}_{2} \mathrm{O}_{4}$ was first determined in 1915 by Bragg [1] and Nishikawa [2]. One unit cell, belonging to the $F d \overline{3} m$ space group, contains eight $\mathrm{AB}_{2} \mathrm{O}_{4}$ units arranged in a pseudo-cubic close-packed (CCP) anion sublattice that contains tetrahedral and octahedral interstices. In a normal II-III structure, one-eighth of the 64 tetrahedral sites are occupied by $\mathrm{A}^{2+}$ cations (Wyckoff equipoint $8 a$ ) and half of the 32 octahedral interstices are taken up by $\mathrm{B}^{3+}$ cations (Wyckoff equipoint $16 d$ for Setting 1 of $F d \overline{3} m$ ). ${ }^{\S}$ One fascinating feature of the spinel structure is its ability to accommodate large amounts of cation disorder. This disordering process leads a wide range of cation distributions with the general formula $\left(\mathrm{A}_{1-}\right.$ $\left.{ }_{x} \mathrm{~B}_{x}\right){ }_{\text {tet }}\left(\mathrm{A}_{x} \mathrm{~B}_{2-x}\right){ }_{\text {oct }} \mathrm{O}_{4}$ (tet and oct represent tetrahedral and octahedral cation sites, respectively). Here $x$ is the inversion parameter, specifying the fraction of B cations occupying tetrahedral sites, with values ranging between 0 (a so-called normal spinel such as $\mathrm{MgAl}_{2} \mathrm{O}_{4}$ : $\left.\left(\mathrm{Mg}_{1}\right)_{\text {tet }}\left(\mathrm{Al}_{2}\right)_{o c t} \mathrm{O}_{4}\right)$ and 1 (an inverse spinel like $\left.\mathrm{MgIn}_{2} \mathrm{O}_{4}:\left(\operatorname{In}_{1}\right)_{\text {tet }}\left(\mathrm{Mg}_{1} \mathrm{In}_{1}\right)_{o c t} \mathrm{O}_{4}\right)$. Many spinel oxides exhibit remarkable electrical, magnetic, and other physical characteristics [3]. Among them, $\mathrm{MgAl}_{2} \mathrm{O}_{4}$ has been considered for potential application as the non-fertile phase in an inert matrix nuclear fuel due to its high radiation tolerance $[4,5]$.

Irradiation-induced structural evolution in many spinel compounds, especially $\mathrm{MgAl}_{2} \mathrm{O}_{4}$, has been extensively studied under various radiation conditions. Although $\mathrm{MgAl}_{2} \mathrm{O}_{4}$ shows excellent amorphization resistance and low volume swelling under ion irradiation at room and elevated temperature [6-9], this compound can be amorphized when irradiated with $400 \mathrm{keV} \mathrm{Xe}$ at cryogenic temperature [10]. Additionally, observation of a cation disordering transition from $x=$ 0 to $x \sim 2 / 3$, (the latter inversion indicating a random spinel) was reported following swift ion

\footnotetext{
${ }^{\S}$ Note that the remaining vacant octahedral and tetrahedral interstices in spinel correspond to the $16 c$ and $8 b / 48 f$ Wyckoff sites, respectively, in Setting 1 of $F d \overline{3} m$. This becomes important later in interpretations of radiationinduced phase transformations.
} 
irradiation [11] and following neutron irradiation [12]. In addition, a phase transformation of $\mathrm{MgAl}_{2} \mathrm{O}_{4}$ from the spinel crystal structure to a disordered rocksalt structure (space group $F m \overline{3} m$ ) has been observed in studies using both low energy ions [13,14] and swift heavy ions [15]. This phase transformation involves not only a mixing of cations, as in the disorder transition, but the movement of cations from tetrahedral to octahedral sites in the crystal lattice. Some experimental and theoretical studies have shown that cation disorder plays a crucial role in controlling radiation tolerance and defect mobility in spinels [10, 16-18]. Therefore, in order to optimize materials for nuclear energy applications, it is important to understand how the presence of cation disorder might affect the formation of metastable phases under radiation environments. However, the irradiation-induced rocksalt transformation in $\mathrm{MgAl}_{2} \mathrm{O}_{4}$ is the subject of some controversy $[11,19]$. This is partly due to the fact that the X-ray atomic form factors for $\mathrm{Mg}$ and $\mathrm{Al}$ are too similar, such that X-ray diffraction (XRD) is not conclusive in identifying the spinelto-rocksalt and order-to-disorder phase transformation.

In this letter, we describe results of ion irradiation damage experiments performed on fullyinverse $\mathrm{MgIn}_{2} \mathrm{O}_{4}$ spinel ( $\left.x \sim 1\right)$, experiments designed to examine radiation-induced cation disorder and concomitant structural evolution of a model spinel compound. To our knowledge, few studies of irradiation stability have been performed on compounds possessing the inverse spinel structure [20, 21]. We performed $400 \mathrm{keV} \mathrm{Ne}$ and $200 \mathrm{keV}$ He ion irradiations on $\mathrm{MgIn}_{2} \mathrm{O}_{4}$ at cryogenic temperature ( $\left.\sim 77 \mathrm{~K}\right)$. Importantly, in contrast to $\mathrm{MgAl}_{2} \mathrm{O}_{4}$, the X-ray atomic form factor differences between $\mathrm{Mg}$ and In allow for more conclusive identification of irradiation-induced phases. Microstructural characterization revealed a phase transformation of $\mathrm{MgIn}_{2} \mathrm{O}_{4}$ from an inverse spinel to a disordered rocksalt structure, following Ne ion irradiation to a peak displacement damage dose of 4 displacements per atom (dpa). At this ballistic damage 
dose, we also observed evidence of partial amorphization of the $\mathrm{MgIn}_{2} \mathrm{O}_{4}$, based on grazing incidence X-ray diffraction (GIXRD) measurements. On the other hand, in the case of He ion irradiations, our study found no evidence for an amorphization transformation or a spinel-torocksalt transformation. However, we did observe a disordering transition of $\mathrm{MgIn}_{2} \mathrm{O}_{4}$ from an inverse $(x \sim 1)$ to a random $(x \sim 2 / 3)$ spinel structure, following He ion irradiation to a dose of 5 dpa. These results indicate that several different structural changes are possible in irradiated spinels. Our study provides new insights into the behavior of complex oxides exposed to energetic ion irradiation conditions.

High purity $\mathrm{MgO}$ and $\mathrm{In}_{2} \mathrm{O}_{3}$ powders from Alfa Aesar (99.9\% purity) were used to produce sintered polycrystalline $\mathrm{MgIn}_{2} \mathrm{O}_{4}$ pellets. X-ray diffraction (XRD) measurements showed the fabricated pellets to be phase pure and in good agreement with published powder diffraction data for $\mathrm{MgIn}_{2} \mathrm{O}_{4}$ spinel with space group $F d \overline{3} m$ (PDF\# 73-2414 [22]).

Ion irradiations were performed at cryogenic temperature $(\sim 77 \mathrm{~K})$ in the Ion Beam Materials Laboratory at Los Alamos National Laboratory, using a Danfysik High Current Research Ion Implanter operating at $200 \mathrm{kV} .400 \mathrm{keV} \mathrm{Ne}^{++}$and $200 \mathrm{keV} \mathrm{He}^{+}$ions were used in this study, in order to assess the effect of different ion species on the radiation damage response of the material.

$\mathrm{MgIn}_{2} \mathrm{O}_{4}$ samples were examined before and after irradiation using GIXRD with a Bruker AXS D8 Advanced X-ray diffractometer Ne ion irradiated samples were also prepared in crosssectional geometries for transmission electron microscopy (TEM) examination using both Philips CM-30 and FEI Tecnai F30 electron microscopes, each operating at $300 \mathrm{kV}$. 
Figure 1(a) shows GIXRD patterns obtained from pristine $\mathrm{MgIn}_{2} \mathrm{O}_{4}$ and $\mathrm{MgIn}_{2} \mathrm{O}_{4}$ irradiated with $200 \mathrm{keV} \mathrm{He}{ }^{+}$ions to fluences of $1 \times 10^{17}$ and $2 \times 10^{17} \mathrm{He} / \mathrm{cm}^{2}$, corresponding to peak displacement damage doses of $~ 2.5$ and 5 dpa (as determined by SRIM [23] calculations, using a displacement threshold energy of $40 \mathrm{eV}$ for all atomic constituents). The first observation in Fig. 1(a) is that in pristine $\mathrm{MgIn}_{2} \mathrm{O}_{4}$, the $\{111\}$ reflection, which is very sensitive to the occupation of the tetrahedral and octahedral sites, is not observed. Structure factor calculations indicate that the extremely weak $\{111\}$ reflection in $\mathrm{MgIn}_{2} \mathrm{O}_{4}$ is an indication of a highly-inverted spinel, $0.9<\mathrm{x}$ $\leq 1.0$, depending on the oxygen dilation parameter, $u$. Thus, we can assume that prior to irradiation, our $\mathrm{MgIn}_{2} \mathrm{O}_{4}$ spinel approximates a fully-inverse spinel, i.e., $\left(\operatorname{In}_{1}\right)_{t e t}\left(\operatorname{In}_{1} \mathrm{Mg}_{1}\right)_{o c t} \mathrm{O}_{4}$.

The GIXRD patterns in Fig. 1(a) reveal that the $\{111\}$ reflection appears upon He ion irradiation, and the $\{111\}$ intensity increases with increasing ion fluence (or dose), while no amorphization is observed. Upon irradiation, atoms are displaced from their initial lattice sites, mixing the cation occupation of the tetrahedral $8 a$ and octahedral $16 d$ sites in the spinel structure, progressing toward a "random" arrangement of $\mathrm{Mg}$ and In cations on $8 a$ and $16 d$ sites. Specifically, for irradiated $\mathrm{MgIn} \mathrm{I}_{4}$, the inverse $\left(\operatorname{In}_{1}\right)_{\text {tet }}\left(\mathrm{Mg}_{1} \operatorname{In}_{1}\right)_{o c t} \mathrm{O}_{4}$ structure transitions toward a random $\left(\mathrm{Mg}_{1-x} \mathrm{In}_{x}\right)_{t e t}\left(\mathrm{Mg}_{x} \mathrm{In}_{2-x}\right)_{o c t} \mathrm{O}_{4}(x \sim 2 / 3)$ cation arrangement. Supplementary Figures 1 and 2 show simulated powder XRD patterns for an inverse $\operatorname{MgIn}_{2} \mathrm{O}_{4}(x=1, u=0.375)$ and a random $\operatorname{MgIn}_{2} \mathrm{O}_{4}$ spinel $(x=2 / 3, u=0.375)$, respectively. The oxygen parameter $u=0.375$ is a hypothetical value for an "ideal" spinel crystal in which all octahedral and tetrahedral lattice interstices are regular. The measured GIXRD patterns from pristine and He irradiated $\mathrm{MgIn}_{2} \mathrm{O}_{4}$ shown in Fig. 1(a) are consistent with the simulated XRD patterns for inverse and random $\mathrm{MgIn}_{2} \mathrm{O}_{4}$ spinel, respectively. These observations indicate that He irradiation in $\mathrm{MgIn}_{2} \mathrm{O}_{4}$ induces a cation disordering transition from an inverse spinel to a random spinel structure. 
Figure 1(b) shows GIXRD patterns obtained from $\mathrm{MgIn}_{2} \mathrm{O}_{4}$ before and after $400 \mathrm{keV} \mathrm{Ne}$ ion irradiations with fluences of $1 \times 10^{15}$ and $1 \times 10^{16} \mathrm{Ne} / \mathrm{cm}^{2}$, corresponding doses of $\sim 0.4$ and $4 \mathrm{dpa}$, respectively. The most important diffraction effect observed following Ne irradiation is that the intensities of the primary spinel peaks (including (220), (311), (422) and (511)) are greatly diminished, while other initially weak reflections such as (222), (400) and (440) become strong in the irradiated spinel. Based on previous studies (see, e.g., Ref. [16]), these observations suggest that Ne ion irradiation is causing more than simple cation disorder on spinel lattice sites; on the other hand, an entirely new phase is being formed in the irradiated $\mathrm{Mg}_{2} \operatorname{In}_{4}$. To determine the crystal structure of this altered crystalline phase, simulated powder XRD patterns were calculated for several structures proposed in earlier studies [19, 24], including cation disordered rocksalt, sphalerite, and antifluorite. The structure factor, $F_{h k l}$ where $h k l$ represents a specific Bragg reflection, was calculated for each model structure. Calculations were based on the kinematical approximation, $F_{h k l}=\Sigma f_{n} \exp \left[2 \pi i\left(h x_{n}+k y_{n}+l z_{n}\right)\right]$, where $f_{n}$ is the atomic form factor for atom $n$ at a fractional coordinate $\left(x_{n}, y_{n}, z_{n}\right)$. Comparing the simulated patterns with those observed in Fig. 1(b), we identified a disordered rocksalt structure as the best fit to the GIXRD observations (XRD simulation shown in Supplementary Figure 3). The new peaks can be indexed as a rocksalt phase with new Miller indices given by $(111)_{\mathrm{R}},(200)_{\mathrm{R}},(220)_{\mathrm{R}},(311)_{\mathrm{R}}$, and $(222)_{\mathrm{R}}\left(\mathrm{R}\right.$ for rocksalt) which correspond to spinel peaks $(222)_{\mathrm{S}},(400)_{\mathrm{S}},(440)_{\mathrm{S}},(622)_{\mathrm{S}}$, and $(444)_{S}$, respectively ( $\mathrm{S}$ for spinel). This new phase is a disordered structure in which the oxygen sublattice maintains a "pseudo" cubic close-packed (CCP) arrangement, as in spinel, while the cations occupy randomly the octahedral lattice interstices, while all tetrahedral interstices are vacant. The repeat unit of the rocksalt phase is half that of spinel; in other words, the lattice 
parameter is reduced from the $\mathrm{MgIn}_{2} \mathrm{O}_{4}$ spinel cubic lattice parameter of $a_{0}^{S}=0.886 \mathrm{~nm}$ to about $a_{0}^{R}=0.443 \mathrm{~nm}$ in the rocksalt phase.

The other significant diffraction effect observed following Ne ion irradiation is a broad diffraction feature that emerges in the scattering angle range, $2 \theta \sim 28-36^{\circ}$. This diffraction feature is interpreted as due to an amorphous phase that forms during ion irradiation. When the Ne irradiation fluence is increased from $1 \times 10^{15}$ to $1 \times 10^{16} \mathrm{ions} / \mathrm{cm}^{2}$, this broad diffraction feature becomes more pronounced. Contrasting this with the He ion irradiation results in Fig. 1(a), Ne ions induce amorphization in $\mathrm{MgIn}_{2} \mathrm{O}_{4}$, while $\mathrm{He}$ ions do not.

Figure 2(a) shows results of cross-sectional transmission electron microscope (XTEM) observations of the microstructure obtained after $\mathrm{Ne}$ ion irradiation of $\mathrm{MgIn}_{2} \mathrm{O}_{4}$ to a fluence of $1 \times 10^{16}$ ions $/ \mathrm{cm}^{2}$. Also shown in Fig. 2(a) are selected area electron diffraction (SAED) patterns obtained from the irradiated layer and the unirradiated substrate. The SAED pattern corresponding to unirradiated substrate is consistent with the $\mathrm{MgIn}_{2} \mathrm{O}_{4}$ spinel structure, aligned along the [110] direction. In the SAED pattern obtained from the irradiated layer, the intensities of the first-order fundamental lattice reflections are reduced significantly and they become diffuse. By contrast, second-order reflections in this same SAED pattern remain strong and sharp. These observations are similar to those obtained in previous radiation studies of spinels $[10,13,14]$, and confirm that $\mathrm{MgIn}_{2} \mathrm{O}_{4}$ transforms to a rocksalt-like structure. The strong, sharp reflections in Fig. 2(a) can be indexed in accordance with a rocksalt unit cell, with half the lattice parameter of the pristine spinel. The presence of the diffuse "spinel" reflections in the SAED pattern from the irradiated layer suggest that the radiation-induced spinel-to-rocksalt phase transformation has not proceeded to completion at the Ne ion dose used in this experiment. 
A high-resolution TEM image obtained from the damage layer is shown in Figure 2(b). This micrograph was obtained with the electron beam oriented along the [110] crystallographic axis with respect to the unirradiated substrate. At least two distinct atomic arrangements, indicated by A and B, are apparent in Fig. 2(b). As compared to the atomic arrangement of region B, the lattice fringe pattern labeled A possesses a doubly-redundant periodicity. Fast Fourier transforms (FFT) of the image contrast obtained from crystallites A and B are in agreement with the SAED patterns in Fig. 2(a) corresponding to the spinel and the metastable rocksalt phases, respectively. In summary, Fig. 2(b) provides additional evidence for a partially-complete, spinel-to-rocksalt phase transformation in $\mathrm{Ne}$ irradiated $\mathrm{MgIn}_{2} \mathrm{O}_{4}$.

It should be noted that our TEM observations (Fig. 2) provide no evidence for a $\mathrm{Ne}$ ion irradiation-induced amorphous phase, as was suggested by our GIXRD observations (Fig. 1(b)). Work is in progress to resolve this paradox. Nevertheless, the focus of our present study is on the spinel-to-rocksalt phase transformation in $\mathrm{MgIn}_{2} \mathrm{O}_{4}$, which we observed by both GIXRD and TEM.

In many previous studies focused on spinel-type materials (primarily on $\mathrm{MgAl}_{2} \mathrm{O}_{4}$ ), an ion irradiation-induced phase transformation from spinel to the rocksalt structure was suggested by electron diffraction analyses. Due to quantitative inaccuracies of electron diffraction and dynamical effects such as multiple scattering, there still exist some ambiguities regarding the true nature of the rocksalt or rocksalt-like variant phase produced in irradiated spinel. The XRD results we have presented here reveal clear evidence for the existence of both a random spinel phase and a rocksalt phase in irradiated inverse $\mathrm{MgIn}_{2} \mathrm{O}_{4}$ spinel. As opposed to previous studies on $\mathrm{MgAl}_{2} \mathrm{O}_{4}$, we have an incredible distinction between the scattering powers of In and $\mathrm{Mg}-$ In 
has an atomic form factor 4 times that of $\mathrm{Mg}$ (this translates to intensity differences up to a factor of 16). Because of this, we can for the first time interrogate the cation occupancy of interstices in a pseudo-ccp anion sublattice. However, it is almost impossible to identify cations on various sites in $\mathrm{MgAl}_{2} \mathrm{O}_{4}$ since $\mathrm{Mg}$ and $\mathrm{Al}$ have almost identical scattering powers. Also, $\mathrm{Mg}$ and $\mathrm{Al}$ have scattering powers very similar to $\mathrm{O}$, so even oxygen sublattice dilations in $\mathrm{MgAl}_{2} \mathrm{O}_{4}$ confuse the cation occupancy. To the best of our knowledge, this is the first experimental evidence where formation of a radiation-induced rocksalt phase in spinel is clearly demonstrated.

In our studies, we observed two distinct effects in $\mathrm{MgIn}_{2} \mathrm{O}_{4}$ following ion irradiation: (1) a cation order-to-disorder (OD) transition from an inverse to a random spinel structure; and (2) a phase transformation from an inverse spinel structure to a rocksalt-like structure. The formation of a random spinel phase is attributed to the process of radiation-induced cation antisite defect formation. On the other hand, the formation of a rocksalt-like phase requires that all of the $\mathrm{Mg}$ and In cations occupy only octahedral sites, leaving all of the tetrahedral interstices vacant. This is achieved via radiation-induced cation Frenkel-pair formation. In fact, cation Frenkels are the defects responsible for the phase transformation from the spinel to the rocksalt structure. Previous molecular dynamics simulations [17] of displacement cascades in $\mathrm{MgIn}_{2} \mathrm{O}_{4}$ showed that radiation-induced cation interstitials preferentially occupy octahedral sites in the inverse spinel $\mathrm{MgIn}_{2} \mathrm{O}_{4}$, while the simulations revealed a corresponding depletion of atoms on tetrahedral ( $8 a$ ) spinel cation sites. Such a process would be consistent with the observed $\mathrm{MgIn}_{2} \mathrm{O}_{4}$ spinel-torocksalt transformation.

We also wish to consider the possibility for ion irradiation "spectrum" effects. Evidence for a spectrum effect might be contained in our observation that $\mathrm{MgIn}_{2} \mathrm{O}_{4}$ only undergoes a spinel-to- 
rocksalt phase transformation when exposed to $400 \mathrm{keV}$ Ne ions (200 keV He ions do not produce such a transformation). This may indicate that structural changes in irradiated $\mathrm{MgIn}_{2} \mathrm{O}_{4}$ are sensitive to details regarding the irradiation spectrum. Figure 3 shows nuclear $(d E / d x)_{n u c l e a r}$ versus electronic $(d E / d x)_{\text {electronic }}$ stopping in $\mathrm{MgIn}_{2} \mathrm{O}_{4}$ for $200 \mathrm{keV} \mathrm{He}$ and $400 \mathrm{keV} \mathrm{Ne}$, calculated using SRIM. It is clear that electronic stopping plays a greater role in the total stopping power for He compared to Ne ions. However, the magnitudes of the electronic stopping powers for He and $\mathrm{Ne}$ are not greatly different near the sample surface (the first $150 \mathrm{~nm}$ or so). Thus, it would be difficult to argue that ionization is responsible for the observed differences in radiation response between $\mathrm{He}$ and $\mathrm{Ne}$ ions. The most noticeable difference among the irradiation conditions used here is the nuclear energy deposition density per incident ion during stopping. Based on SRIM calculation, the nuclear stopping power exhibits a maximum of $\sim 0.3$ $\mathrm{keV} / \mathrm{nm} / \mathrm{ion}$ for $400 \mathrm{keV} \mathrm{Ne}$, which is much greater than that for He ions (less than 0.02 $\mathrm{keV} / \mathrm{nm} / \mathrm{ion}$ over the entire ion range). In other words, per ion, Ne produces more dense point defect cascades, while He produces widely separated (isolated) point defects. This means that He irradiation will result in much lower cascade defect densities compared to Ne irradiation.

Nevertheless, we should be able to establish similar retained defect concentrations for He versus Ne by increasing the He ion fluence. However, we found that even when our He ion displacement damage dose exceeded that of $\mathrm{Ne}$, the concentration of surviving defects for He was still far less than for Ne. In fact, the only retained defects in the case of He are cation antisites, while for $\mathrm{Ne}$, even at the lowest dose examined in this study, both cation antisites and Frenkels appear to survive (in the rocksalt phase) and additional defects must be associated with the amorphization transformation. The more dense Ne cascades produce damage structures wherein cation Frenkel pairs are retained in substantial concentrations (with a bias toward $16 c-8 a$ 
and $16 c-16 d$ interstitial-vacancy Frenkel pairs (i.e., $8 b$ and $48 f$ spinel interstitials do not survive)). For He, cation antisite defects survive while cation Frenkel pairs succumb to annihilation. Our results seem to suggest that damage efficiency is greater for the denser $\mathrm{Ne}$ cascades compared to dilute He ones. This is contrary to observations in monatomic metals [25]. Nevertheless, our results are consistent with previous observations showing that, depending on the irradiation spectrum, spinel experiences either a cation OD transition $[11,14,26]$ or spinel undergoes transformation to either a rocksalt or amorphous phase $[10,13]$.

\section{Acknowledgements}

This work was sponsored by the Department of Energy, Office of Basic Energy Sciences, Division of Materials Sciences and Engineering. Los Alamos National Laboratory, an affirmative action equal opportunity employer, is operated by Los Alamos National Security, LLC, for the National Nuclear Security Administration of the US DOE under contract DEAC52-06NA25396. We thank Professor Karl Whittle for useful discussions. 


\section{Reference}

[1] W. H. Bragg, Philos. Mag., 30(176) (1915) 305-315.

[2] S. Nishikawa, Proc. Math. Phys. Soc. Tokyo, 8 (1915) 199-209.

[3] N. W. Grimes, Phys. Technol., 6 (1975) 22-27.

[4] H. Matzke, V. V. Rondinella, and T. Wiss, J. Nucl. Mater. 274 (1999) 47-53.

[5] T. Wiss and H. Matzke, Radiat. Meas. 31 (1999) 507-514.

[6] L. W. Hobbs, F. W. Clinard, Jr., S. J. Zinkle, and R. C. Ewing, J. Nucl. Mater. 216 (1994) 291- 321 .

[7] F. W. Clinard, Jr., G. F. Hurley, L. W. Hobbs, D. L. Rohr, and R. A. Youngman, J. Nucle. Mater. 122 \& 123 (1984) 1386-1392.

[8] C. Kinoshita, S. Matsamura, K. Yasuda, T. Soeda, M. Noujima, Mat. Res. Soc. Symp. Proc. 540 (1999) 287- 297.

[9] S. J. Zinkle, J. Am. Ceram. Soc. 72 (1989) 1343-1351.

[10] N. Yu, K. E. Sickafus, Michael Nastasi, Philos. Mag. Lett., 70 (1994) 235-240.

[11] D. Simeone, C. Dodane-Thiriet, D. Gosset, P. Daniel, M. Beauvy, J. Nucl. Mater. 300 (2002) 151-160.

[12] K. E. Sickafus, A. C. Larson, N. Yu, M. Nastasi, G. W. Hollenberg, F. A. Garner, R. C. Bradt, J. Nucl. Mater. 219 (1995) 128-134.

[13] M. Ishimaru, I. Afanasev-Charkin, K. Sickafus, Appl. Phys. Lett. 76-18 (2000) 25562558.

[14] K. E. Sickafus, N. Yu, R. Devanathan, M. Nastasi, Nucl. Instrum. And Meth. B 106 (1995) 573-578. 
[15] K. Yasuda, T. Yamamoto, M. Shimada, S. Matsumura, Y. Chimi, N. Ishikawa, Nucl. Instr. And Meth. Phys. Res. B 250 (2006) 238-244.

[16] B. P. Uberuaga, M. Tang, C. Jiang, J. A. Valdez, R. Smith, Y. Wang, K. E. Sickafus, Nat. Commun. 6 (2015) 8750.

[17] D. Bacorisen, R. Smith, B. P. Uberuaga, K. E. Sickafus, J. A. Ball, R. W. Grimes, Phys. Rev. B 74 (2006) 214105.

[18] C. Jiang, K. E. Sickafus, C. R. Stanek, S. P. Rudin, and B. P. Uberuaga, Phys. Rev. B 86 (2012) 024203.

[19] K. E. Sickafus, J. Nucl. Mater. 312 (2003) 111-123.

[20] L. Wang, W. Gong, S. Wang, and R. C. Ewing, J. Am. Ceram. Soc. 82 [12] (1999) 332129.

[21] P. Xu, M. Tang, J. C. Nino, J. Nucl. Mater. 389 (2009) 410-415.

[22] International Centre for Diffraction Data, Powder Diffraction File, Joint Committee on Powder Diffraction Standards, Philadelphia, PA, 1974-present.

[23] J. F. Ziegler, J. P. Biersack, U. Littmark, The Stopping and Range of Ions in Solids, (Pergamon, New York, 1985).

[24] R. Devanathan, K. E. Sickafus, N. Y, M. Nastasi, Philos. Mag. Lett. 72 (1995) 155-161.

[25] R. S. Averback, R. Benedek, K. L. Merkle, J. Nucl. Mater. 75 (1978) 162-166.

[26] G. Baldinozzi, D. Simeone, D. Gosset, S. Surble, L. Mazerolles, L. Thome, Nucl. Inst. And Meth. Nucl. Phys. B 266 (2008) 2848-2853. 


\section{Figure Captions}

Figure 1

(a) GIXRD patterns obtained from pristine $\mathrm{MgIn}_{2} \mathrm{O}_{4}$ and $\mathrm{MgIn}_{2} \mathrm{O}_{4}$ irradiated with $200 \mathrm{keV} \mathrm{He}^{+}$ ions to fluences of $1 \times 10^{17}$ and $2 \times 10^{17} \mathrm{He} / \mathrm{cm}^{2}$, corresponding to peak displacement damage doses of $\sim 2.5$ and 5 dpa. $\{$ hkl $\}$ reflection subscript, $S$, represents the spinel phase.

(b) GIXRD patterns obtained from pristine $\mathrm{MgIn}_{2} \mathrm{O}_{4}$ and $\mathrm{MgIn}_{2} \mathrm{O}_{4}$ irradiated with $400 \mathrm{keV} \mathrm{Ne}$ ions to fluences of $1 \times 10^{15}$ and $1 \times 10^{16} \mathrm{Ne} / \mathrm{cm}^{2}$, corresponding to peak displacement damage doses of $\sim 0.4$ and 4 dpa. $\{h k l\}$ reflection subscripts, $S$ and $R$, represent the spinel and rocksalt phases, respectively.

Figure 2

(a) Cross-sectional TEM bright-field image (left) obtained from $\mathrm{MgIn}_{2} \mathrm{O}_{4}$ irradiated to a fluence of $1 \times 10^{16} \mathrm{Ne} / \mathrm{cm}^{2}$ and corresponding selected area electron diffraction (SAED) patterns (right) obtained from the irradiated layer (upper) and the unirradiated substrate (lower). The SAED pattern obtained from the pristine (unirradiated) sample region shows strong $\{002\}_{S}$ and $\{111\}_{S^{-}}$ type reflections, which is contrary to the structure factor for inverse $\mathrm{MgIn}_{2} \mathrm{O}_{4}$ spinel. The reason for this anomaly is double diffraction (see Supplementary Fig. 4 caption for further discussion).

(b) HRTEM image obtained from the irradiated layer. Also shown as insets are FFT diffractograms from the regions labeled A and B in the HRTEM image.

Figure 3

Comparison of electronic versus nuclear stopping for $200 \mathrm{keV} \mathrm{He}$ and $400 \mathrm{keV} \mathrm{Ne}$ ions in $\mathrm{MgIn}_{2} \mathrm{O}_{4}$. Electronic energy loss $(d E / d x)_{\text {electronic }}$ and nuclear energy loss $(d E / d x)_{n u c l e a r}$ were calculated using SRIM [23]. 


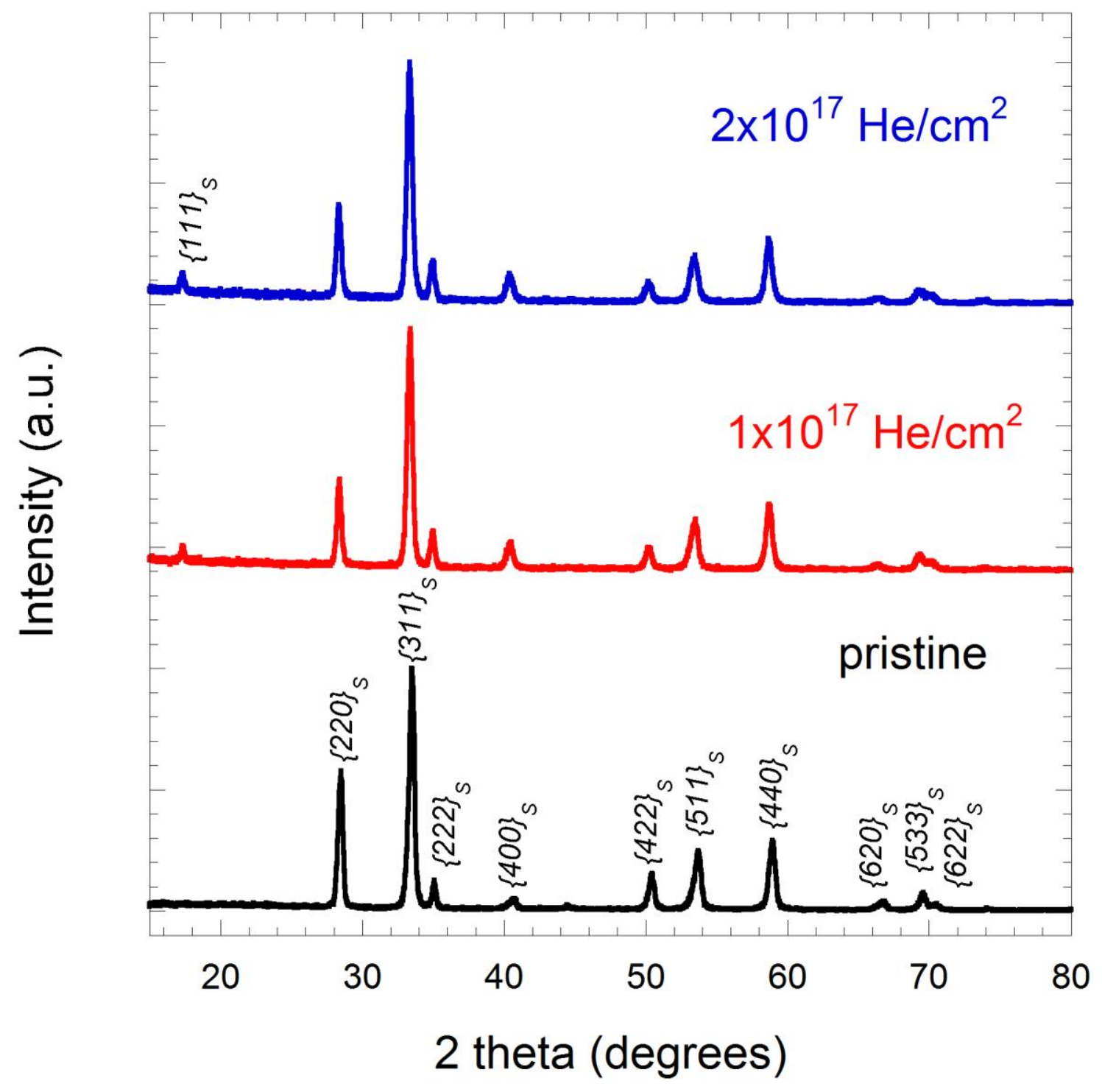

Figure 1a 


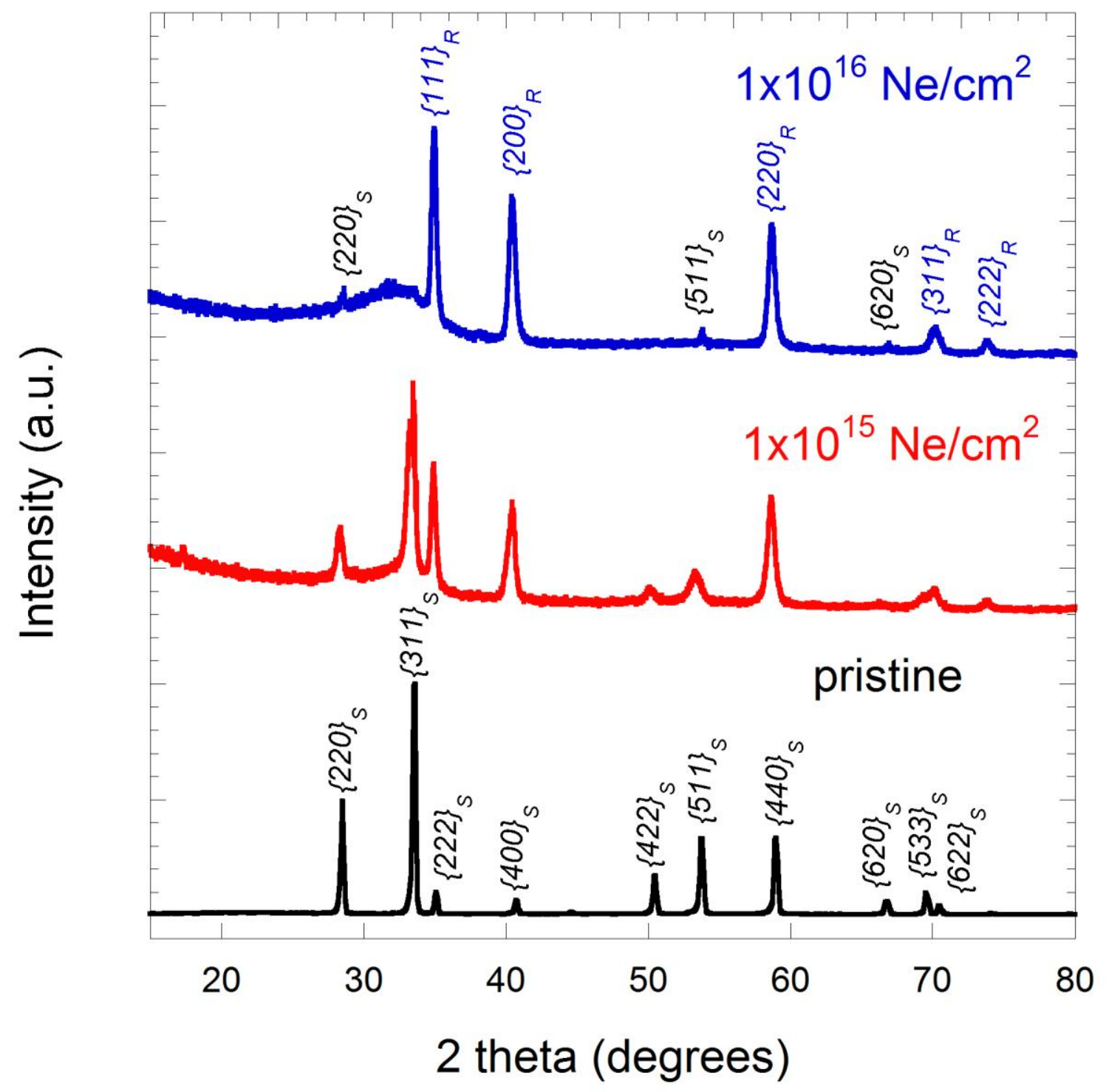

Figure 1b 


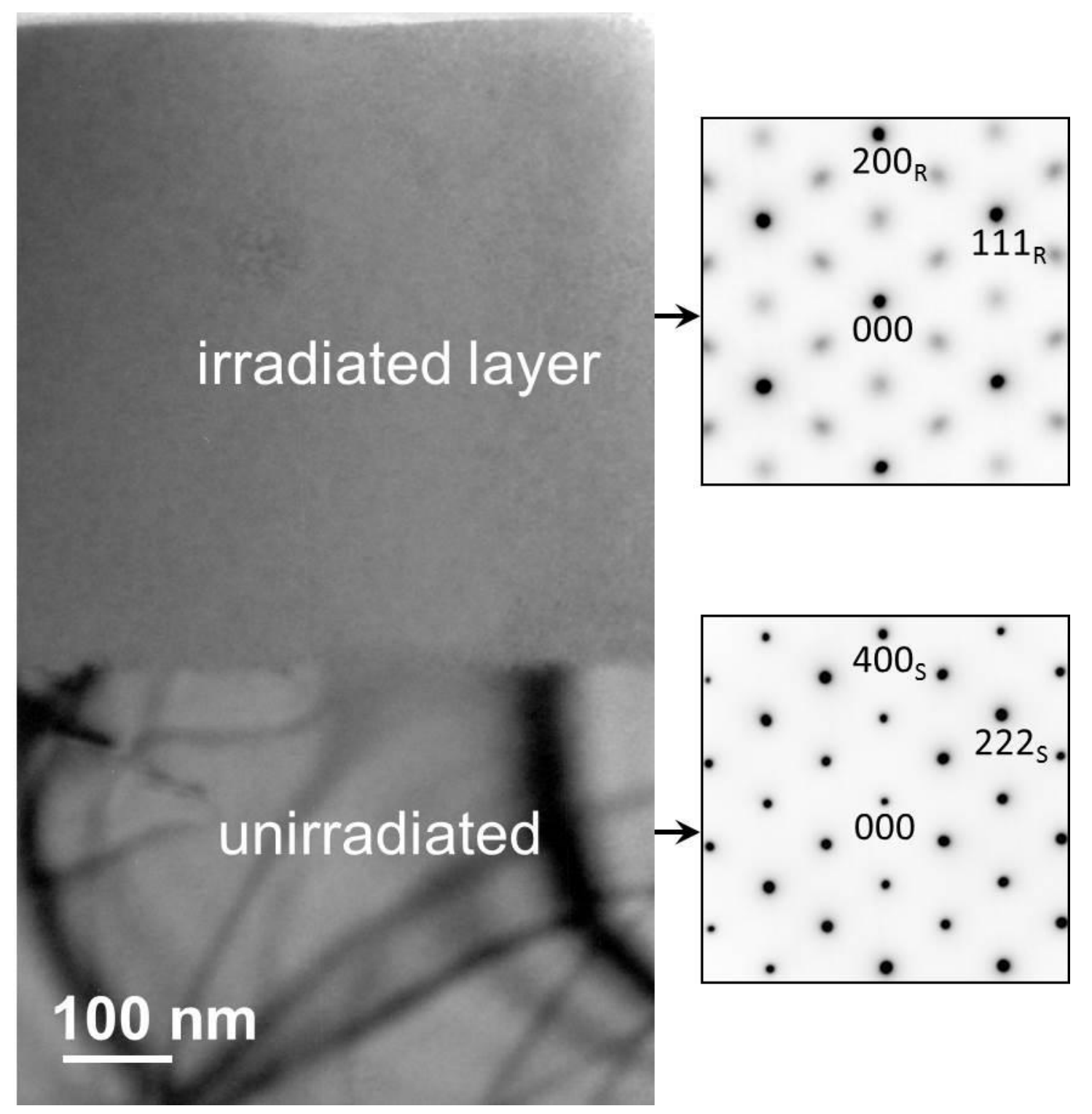

Figure 2 


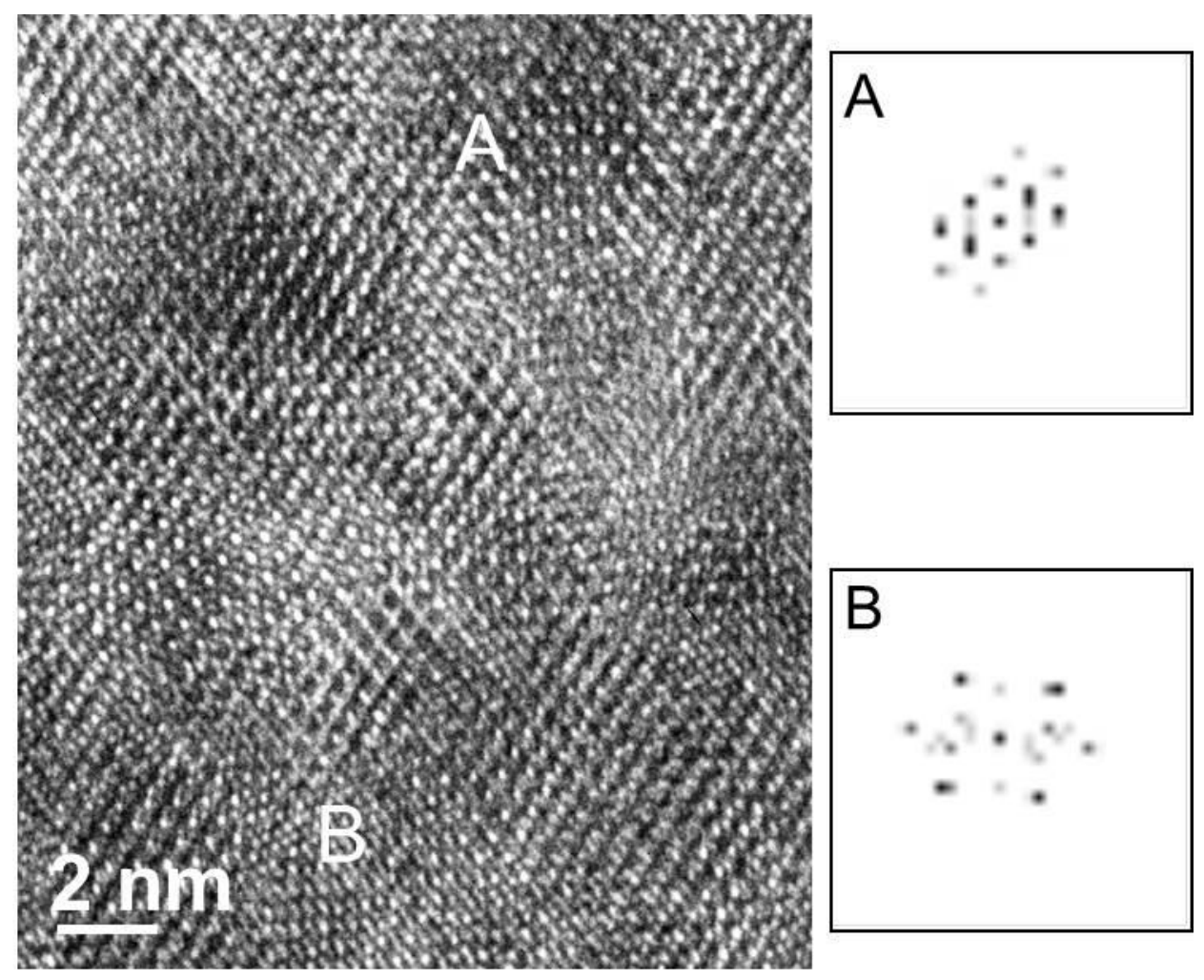

Figure 3 


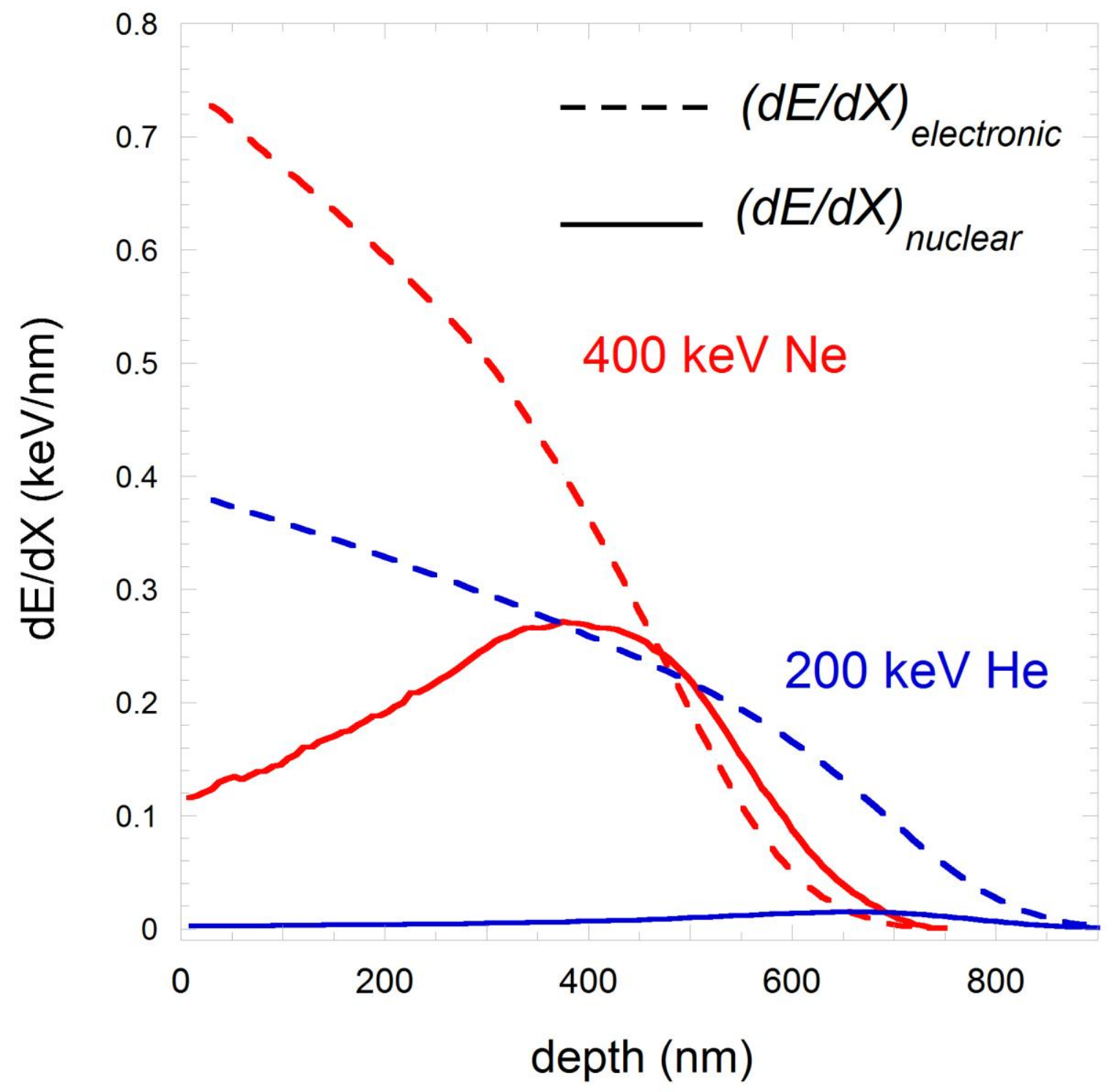

Figure 4 
Graphical Abstract
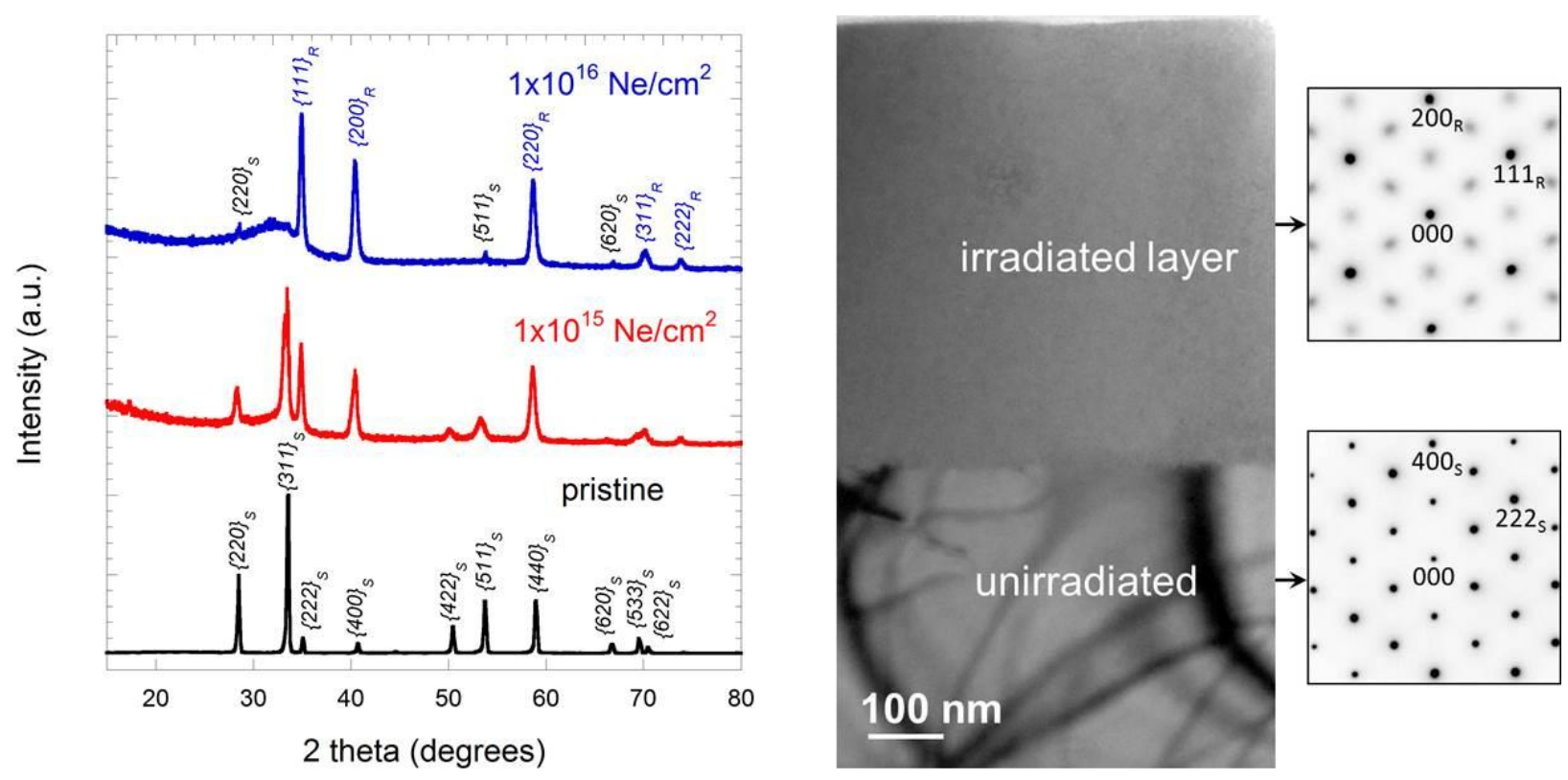